\title{
Cost-Effective Paramagnetic Bead Technique for Purification of Cycle Sequencing Products
}

\author{
Slavica Mijatovic-Rustempasic, ${ }^{1}$ Michael A. Frace, ${ }^{2}$ and Michael D. Bowen ${ }^{1}$ \\ ${ }^{1}$ Gastroenteritis and Respiratory Viruses Laboratory Branch, Division of Viral Diseases, National Center for Immunization \\ and Respiratory Diseases, Centers for Disease Control and Prevention, 1600 Clifton Road, Atlanta, GA 30333, USA \\ ${ }^{2}$ Biotechnology Core Facility Branch, Division of Scientific Resources, National Center for Emerging and Zoonotic Infectious Diseases, \\ Centers for Disease Control and Prevention, 1600 Clifton Road, Atlanta, GA 30333, USA \\ Correspondence should be addressed to Michael D. Bowen, mkb6@cdc.gov
}

Received 1 June 2012; Revised 23 August 2012; Accepted 29 August 2012

Academic Editor: Jose M. Requena

Copyright ( 2012 Slavica Mijatovic-Rustempasic et al. This is an open access article distributed under the Creative Commons Attribution License, which permits unrestricted use, distribution, and reproduction in any medium, provided the original work is properly cited.

\begin{abstract}
The quality of sequencing results depends greatly upon the quality and purity of the template as well as the purity of the fluorescently labeled products generated by cycle sequencing. Numerous approaches have been used for purification of cycle sequencing products, including alcohol precipitation, affinity-based chromatography, size exclusion chromatography, commercially-available proprietary methods, and paramagnetic bead technology. In this paper, we describe an affordable paramagnetic technology method using BioMag Carboxyl beads. Compared to other well-established, proprietary methods for purification of cycle sequencing products, this method produced consistently good results, with a very low reagent cost and short procedure time.
\end{abstract}

\section{Introduction}

Since the 1990's, the most widely used method of automated DNA sequencing has been the Sanger dye-termination enzymatic sequencing method, used in conjunction with gelbased or capillary array electrophoresis sequencers that use laser-induced fluorescence detection [1]. Although nextgeneration sequencing technologies [2,3] are quickly gaining ground, Sanger sequencing remains the method of choice for many researchers for sequencing PCR amplicons and for filling in sequence gaps in genomic sequencing projects. For Sanger sequencing, most laboratories use BigDye Terminator v.3.1 Cycle Sequencing kits (Applied Biosystems, Foster City, CA, USA).

Two major factors influence the final quality of raw sequence data: (1) concentration, integrity, and purity of the DNA template, and (2) the effective removal of excess dye terminators and impurities from cycle sequenced products before analysis on sequencing instruments $[4,5]$. Methods for purification of cycle sequencing products often can be costly, time-consuming, or both, and may result in partial or full loss of the original template. The choice of technique used for purification of cycle sequencing products can depend on several factors such as cost, hands-on time, need for high-throughput processing, sequence data quality, and the preference of the users. Numerous approaches are available for the purification of cycle sequencing products, including as alcohol precipitation [6], affinity-based chromatography [7], size exclusion chromatography techniques [8-10], proprietary kits, and use of the paramagnetic particles or beads $[11,12]$.

The use of magnetic beads for purification of cycle sequencing products was introduced in 1992 [12]. In this method, purification relies upon adsorption of DNA to magnetic beads in the presence of ethanol. Bead-bound cycle sequencing products are washed with ethanol and DNA is eluted off the magnetically-captured beads in water or other low ionic solutions. Several commercial magnetic beads kits for purification of cycle sequenced products became available in the last 10 years including the CleanSEQ (Agencourt Bioscience Corporation, Beverly, MA, USA), MagDTR Magnetic Resin (Edge Biosystems, Gaithersburg, MD, USA), MagSi-DNA clean ${ }^{\text {FIX }}$ (MagnaMedics, Geleen, Netherlands), 
and Wizard MagneSil Sequencing Reaction Clean-Up System (Promega, Madison, WI, USA). Although these kits are convenient and typically perform well, their cost is high; the price per sample ranges from US $\$ 0.25$ to $\$ 0.57$. A low-cost alternative method, alcohol precipitation, requires a high degree of technical proficiency to yield consistently good quality data. Proprietary kits employing affinity or sizeexclusion chromatography methods typically yield excellent results but are expensive. We have developed a method which uses inexpensive magnetic beads (BioMag Carboxyl beads, Bangs Laboratories, Inc., Fishers, IN, USA) at the fraction of the cost of the commercially available magnetic beads, kits (less than US $\$ 0.02$ per sample). The method is fast ( $25 \mathrm{~min}$ per 96 samples) and has minimal potential for user error. In this paper, we present our method and demonstrate that the quality of sequence data obtained is comparable to that obtained using more expensive proprietary methods.

\section{Materials and Methods}

2.1. Preparation of BioMag Carboxyl Beads. Prior to use, the BioMag Carboxyl beads must be washed and resuspended in $5 \mathrm{M} \mathrm{NaCl}$. Beads from a $1 \mathrm{~mL}$ volume of bead stock were captured using a $1.5 \mathrm{~mL}$ magnetic separator (Bangs Laboratories, Inc., Fishers, IN, USA) and then washed in $1 \mathrm{~mL}$ of $0.5 \mathrm{M}$ EDTA. The beads were captured again using a magnetic separator and resuspended in $0.5 \mathrm{~mL}$ of $0.5 \mathrm{M}$ EDTA. The bead suspension was then combined with $19.5 \mathrm{~mL}$ of $5.0 \mathrm{M} \mathrm{NaCl}$. The washed bead suspension has a shelf life of 12 months when stored at $4{ }^{\circ} \mathrm{C}$.

\subsection{Purification of Cycle Sequencing Products Using BioMag} Carboxyl Beads. Amplicons of rotavirus VP4 and VP7 genes were generated by reverse transcription-polymerase chain reaction (RT-PCR) amplification of viral RNA as described [13-15]. Cycle sequencing was performed using BigDye Terminator v.3.1 Cycle Sequencing mix (10 or $20 \mu \mathrm{L}$ reactions) in a 96-well reaction plate. Ten microliters of well-mixed bead suspension was added to each cycle sequencing reaction along with $42 \mu \mathrm{L}$ ( $10 \mu \mathrm{L}$ reaction) or $62 \mu \mathrm{L}$ ( $20 \mu \mathrm{L}$ reaction) of $85 \%$ ethanol and mixed by pipetting up and down. The reaction plate was inserted onto a SPRIPlate 96R ring magnetic plate (Agencourt Bioscience Corp., Beverly, MA, USA) and incubated for 5 minutes at room temperature. The reaction plate/magnetic plate assembly was then inverted onto a lintfree wipe and tapped gently a few times to remove any residual liquid. The reaction plate/magnetic plate assembly was then returned to an upright position and $200 \mu \mathrm{L}$ of $85 \%$ ethanol was added into each well. After allowing the beads to settle for $30 \mathrm{sec}$, the reaction plate/magnetic plate assembly was inverted and tapped gently onto a lint-free wipe to remove any residual liquid. The $85 \%$ ethanol wash was then repeated once ( 2 washes total). The reaction plate/magnetic plate assembly was then inverted onto a lint-free wipe and centrifuged at $65 \times \mathrm{g}$ for $10 \mathrm{sec}$. The reaction plate/magnetic plate assembly was returned to an upright position and $40 \mu \mathrm{L}$ of molecular biology grade water was added to each well. After placing a plate septum onto the 96 -well reaction plate, it was removed from the SPRIPlate 96R ring magnetic plate and placed into a 96-well plate base into which a Direct Inject Magnet (Agencourt Bioscience Corp., Beverly, MA, USA) had been inserted. After applying a plate retainer, the reaction plate assembly was installed on an ABI Prism 3130xl Genetic Analyzer (Applied Biosystems, Foster City, CA, USA), and sequencing products were analyzed using 3130 POP-7 Performance Optimized Polymer and $50 \mathrm{~cm}$ 3130xl and 3100 Capillary Array (Applied Biosystems, Foster City, CA, USA).

\subsection{Production of Replicate Sequences Using Other Cycle} Sequencing Purification Methods. Sequences obtained by using the paramagnetic bead technique were compared with those obtained when cycle sequencing products were purified by (1) alcohol precipitation; (2) a widely-used, proprietary centrifugal size-exclusion column designed to remove unincorporated dideoxynucleotide terminators from cycle sequencing reactions; (3) a proprietary in-well purification kit which captures unwanted cycle sequencing reaction components and then collects them at the bottom of each well through a centrifugation step. Amplicons of rotavirus VP4 and VP7 genes were generated by reverse transcriptionpolymerase chain reaction (RT-PCR) amplification of viral RNA and then subjected to cycle sequencing as described. For each of the purification techniques, three $10 \mu \mathrm{L}$ volumes of cycle sequencing reaction were purified in triplicate using each technique. Purified cycle sequencing products were analyzed on an ABI Prism 3130xl Genetic Analyzer as described.

\section{Results and Discussion}

Cycle sequencing using BigDye Terminator v.3.1 Cycle Sequencing mix was carried out using $38-86$ ng of RT-PCR product per reaction. Sequences obtained after purification of the cycle sequencing product with size exclusion columns, the in-well method, and the BioMag Carboxyl bead method averaged more than $800 \mathrm{bp}$ of high quality ( $>95 \%$ ) sequence (Table 1). The chromatography peaks were well resolved and symmetrical, and the peak height was consistent for all 3 methods (Figure 1). Use of the size exclusion column and in-well methods resulted in the best overall sequence quality and read length. The length of sequences produced using the BioMag Carboxyl beads were similar, and the quality of sequences was slightly lower but within the standard deviation ranges of the size-exclusion column and in-well methods (Table 1). Dye blobs were noted around base no. 70 in both the in-well and bead methods (Figure 1). While this artifact is seen occasionally in sequences prepared using this bead cleanup method (as well as the in-well method), the sequence typically can be read accurately in this region. The two main advantages of BioMag Carboxyl beads compared to the 2 proprietary methods are much lower cost per sample and reduced time necessary to prepare the fluorescent products for sequencing (Table 1). The alcohol precipitation method has the lowest cost per sample (Table 1) but yielded the poorest quality sequences (Table 1, Figure 1). We found that this method is very prone to user error when a 96- 
TABLE 1: Summary of results of cycle sequencing purification methods examined in this study.

\begin{tabular}{|c|c|c|c|c|c|}
\hline Technique & $\begin{array}{c}\text { Sequence } \\
\text { length/quality }\end{array}$ & $\begin{array}{l}\text { Cost, US } \$ \text { b } \\
\text { (per sample) }\end{array}$ & $\begin{array}{c}\text { Time needed } \\
\text { (per } 96 \text { samples) }\end{array}$ & Advantages & Disadvantages \\
\hline BioMag Carboxyl beads & $815 \pm 1 / 97 \pm 3$ & $0.006-0.015$ & $25 \mathrm{~min}$ & $\begin{array}{l}\text { Low cost } \\
\text { Quick } \\
\text { Good results }\end{array}$ & $\begin{array}{l}\text { Moderate potential } \\
\text { for user error } \\
\text { Occasional blobs }\end{array}$ \\
\hline $\begin{array}{l}\text { Proprietary size exclusion } \\
\text { columns }\end{array}$ & $816 \pm 1 / 99 \pm 1$ & $2.2-2.6$ & $3-4 h$ & Best results & $\begin{array}{c}\text { Costly } \\
\text { Time consuming } \\
\text { Moderate potential } \\
\text { for user error }\end{array}$ \\
\hline $\begin{array}{l}\text { Proprietary } \\
\text { in-well } \\
\text { technique }\end{array}$ & $816 \pm 1 / 99 \pm 1^{*}$ & 1.94 & $37 \mathrm{~min}$ & $\begin{array}{c}\text { Quick } \\
\text { Good results } \\
\text { Low potential for user } \\
\text { error }\end{array}$ & $\begin{array}{c}\text { Costly } \\
\text { Short shelf life } \\
\text { Occasional blobs }\end{array}$ \\
\hline Ethanol precipitation & $744 \pm 35 / 87 \pm 11^{* *}$ & $\sim 0.001$ & $1 \mathrm{~h} 45 \mathrm{~min}$ & Lowest cost & $\begin{array}{c}\text { Loss of amplicon } \\
\text { Low quality results } \\
\text { High potential for } \\
\text { user error }\end{array}$ \\
\hline
\end{tabular}

${ }^{a}$ Sequence length and quality scores for overlapping fragments were obtained directly from Sequencher 4.8 software (Gene Codes Corporation, Inc., Ann Arbor, MI). Prior to analyzing the data, raw sequences were trimmed by aligning all of the sequences of one reaction and determining the unusable part of the longest sequence and cutting all sequences at that base. Measurements and standard deviation are calculated using triplicate results of 4 different reactions (primers used were con2, con3, VP7R and $9 \operatorname{con} 1 \mathrm{~L}$ ). Measurement based on $* 1$ and $* * 2$ fewer sequences due to low quality of final sequence.

${ }^{\mathrm{b}}$ Cost per reaction was calculated by totaling the cost of consumables needed to clean one $10 \mu \mathrm{L}$ cycle sequencing reaction and does not account for the cost of equipment.

${ }^{\mathrm{c}}$ Time needed to purify 96 samples was measured from the moment the plate containing the cycle sequencing reactions was removed from the thermocycler after cycle sequencing to the time purification was completed and the 96 -well septum was placed on the plate.

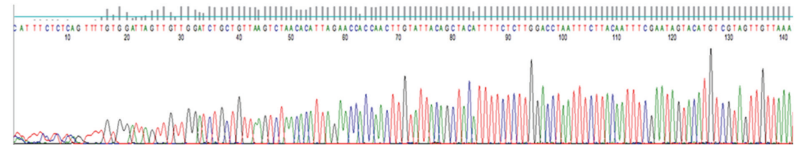

(a)

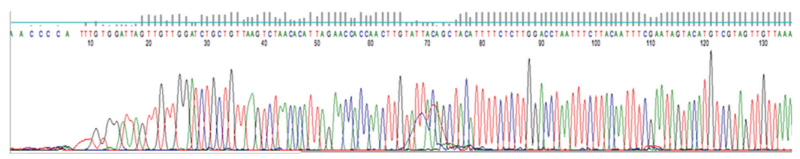

(c)

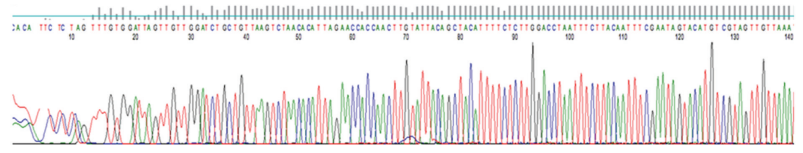

(b)

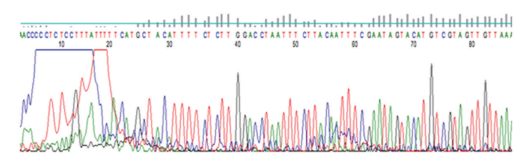

(d)

FIGURE 1: Representative chromatograms of the rotavirus Wa strain VP7 gene sequenced with VP7R primer and purified by proprietary size-exclusion columns (a), a proprietary in-well clean up kit (b), BioMag Carboxyl beads (c), and alcohol precipitation (d).

well plate is used, and the quality of sequencing results was highly dependent upon the user's technical skill. The potential for user error was lowest for the BioMag Carboxyl beads technique and the proprietary size exclusion columns. An automated version of BioMag Carboxyl beads technique has been used successfully to clean up hundreds of thousands of cycle sequencing reactions using both PCR products (500$15,000 \mathrm{bp}$ in length generated using multiple brands of PCR enzymes) as well as plasmids. Sequences obtained using reactions cleaned up by this method have been analyzed on both the ABI Prism 3130xl Genetic Analyzer and the AB 3730xl DNA Analyzer.

In conclusion, BioMag Carboxyl beads produce results comparable to size-exclusion columns and the proprietary kit we tested, and were superior to alcohol precipitation.
Cost is a clear advantage, with the BioMag Carboxyl bead technique being up to 200 times less expensive than the proprietary methods we evaluated and 20-40 times less expensive than other commercially available magnetic bead kits. BioMag Carboxyl bead purification offers the highest quality sequence at the lowest reagent cost and short procedure time, although there is an initial investment in reusable hardware.

\section{Disclaimer}

The findings and conclusions in this paper are those of the authors and do not necessarily represent the official position of the Centers for Disease Control and Prevention. Names of specific vendors, manufacturers, or products are included 
for public health and informational purposes; inclusion does not imply endorsement of the vendors, manufacturers, or products by the Centers for Disease Control and Prevention or the US Department of Health and Human Services.

\section{Conflict of Interests}

The authors declare that they have no conflict of interests.

\section{Acknowledgment}

The authors would like to thank Krisztian Banyai, Nicole Gregoricus, Kimberly R. Foytich, and Osbourne Quaye for their assistance with this study. They also wish to thank Mary McCauley for editorial assistance.

\section{References}

[1] R. A. Mathies and X. C. Huang, "Capillary array electrophoresis: an approach to high-speed, high-throughput DNA sequencing," Nature, vol. 359, pp. 167-169, 1992.

[2] N. Rusk, "Torrents of sequence," Nature Methods, vol. 8, no. 1, p. 44, 2011.

[3] M. Margulies, M. Egholm, W. E. Altman et al., "Genome sequencing in microfabricated high-density picolitre reactors," Nature, vol. 437, no. 7057, pp. 376-380, 2005.

[4] X. Tong and L. M. Smith, "Solid-phase method for the purification of DNA sequencing reactions," Analytical Chemistry, vol. 64, no. 22, pp. 2672-2677, 1992.

[5] X. Tong and L. M. Smith, "Solid phase purification in automated DNA sequencing," DNA Sequence, vol. 4, no. 3, pp. 151$162,1993$.

[6] J. Sambrook, D. Russell, and eds, Eds., Molecular Cloning: A Laboratory Manual, Cold Sring Harbor Laboratory Press, New York, NY, USA, 3rd edition, 2001.

[7] W. Warren and J. Doniger, "HPLC purification of polymerase chain reaction products for direct sequencing," BioTechniques, vol. 10, no. 2, pp. 216-220, 1991.

[8] O. Salas-Solano, M. C. Ruiz-Martinez, E. Carrilho, L. Kotler, and B. L. Karger, "A sample purification method for rugged and high-performance DNA sequencing by capillary electrophoresis using replaceable polymer solutions. B. Quantitative determination of the role of sample matrix components on sequencing analysis," Analytical Chemistry, vol. 70, no. 8, pp. 1528-1535, 1998.

[9] M. C. Ruiz-Martinez, O. Salas-Solano, E. Carrilho, L. Kotler, and B. L. Karger, "A sample purification method for rugged and high-performance DNA sequencing by capillary electrophoresis using replaceable polymer solutions. A. Development of the cleanup protocol," Analytical Chemistry, vol. 70, no. 8, pp. 1516-1527, 1998.

[10] R. R. Pogue, M. E. Cook, L. R. Livingstone, and S. W. Hunt III, "Preparation of template for automated sequencing using QIAGEN resin,” BioTechniques, vol. 15, no. 3, pp. 376-381, 1993.

[11] C. J. Elkin, H. Kapur, T. Smith et al., "Magnetic bead purification of Labeled DNA fragments for high-throughput capillary electrophoresis sequencing," BioTechniques, vol. 32, no. 6, pp. 1296-1300, 2002.

[12] G. Fry, E. Lachenmeier, E. Mayrand et al., "A new approach to template purification for sequencing applications using paramagnetic particles," BioTechniques, vol. 13, no. 1, pp. 124131, 1992.

[13] B. K. Das, J. R. Gentsch, H. G. Cicirello et al., "Characterization of rotavirus strains from newborns in New Delhi, India," Journal of Clinical Microbiology, vol. 32, no. 7, pp. 1820-1822, 1994.

[14] J. R. Gentsch, R. I. Glass, P. Woods et al., "Identification of group A rotavirus gene 4 types by polymerase chain reaction," Journal of Clinical Microbiology, vol. 30, no. 6, pp. 1365-1373, 1992.

[15] M. Iturriza-Gómara, B. Isherwood, U. Desselberger, and J. Gray, "Reassortment in vivo: driving force for diversity of human rotavirus strains isolated in the United Kingdom between 1995 and 1999," Journal of Virology, vol. 75, no. 8, pp. 36963705, 2001. 


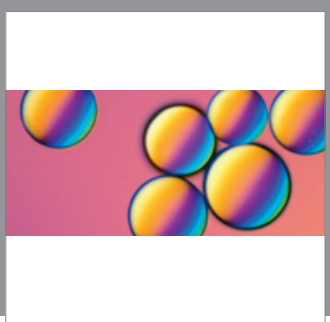

Peptides

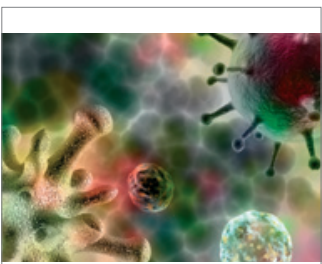

\section{Virology}

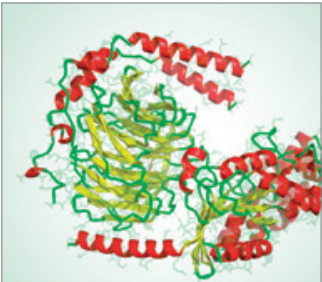

Signal Transduction
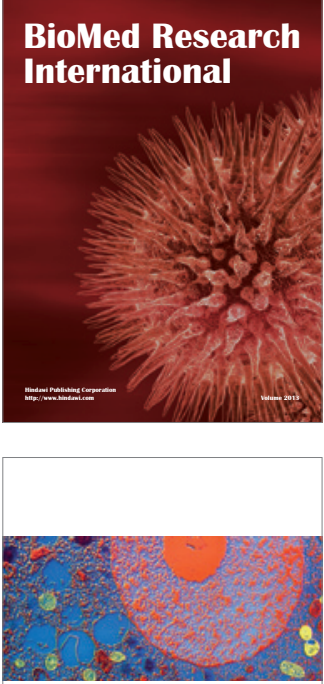

ISRN

Cell Biology
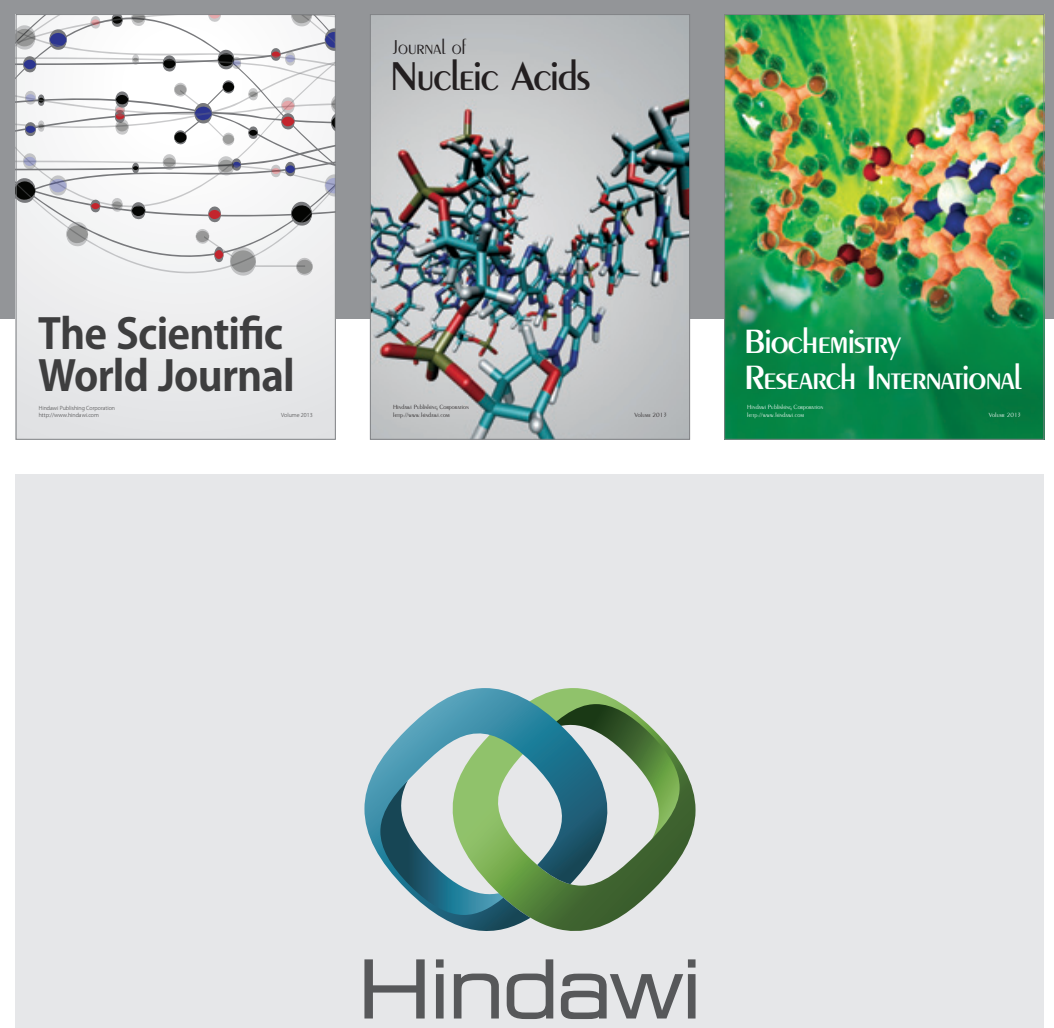

Submit your manuscripts at http://www.hindawi.com

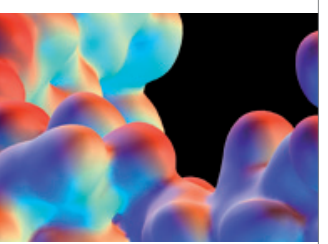

Enzyme Research

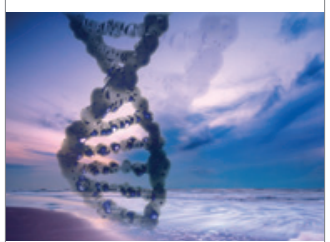

Evolutionary Biology
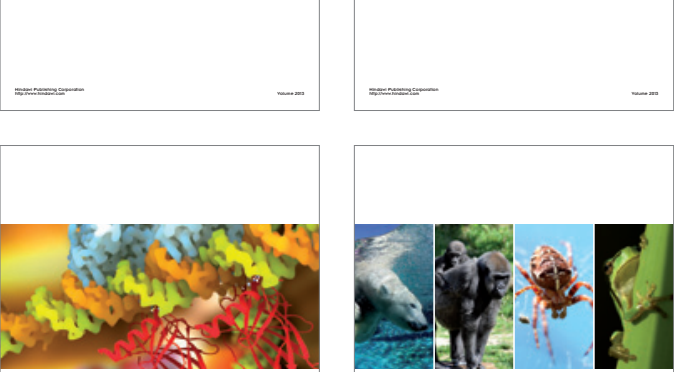

ISRN

Molecular Biology

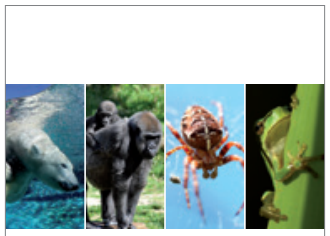

ISRN Zoology

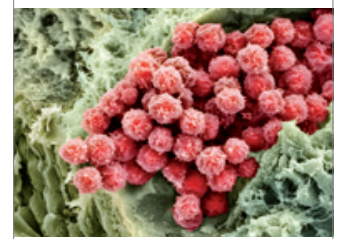

Stem Cells International

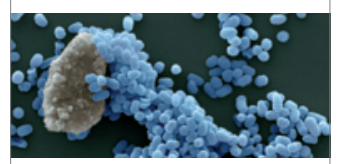

ISRN

Biotechnology
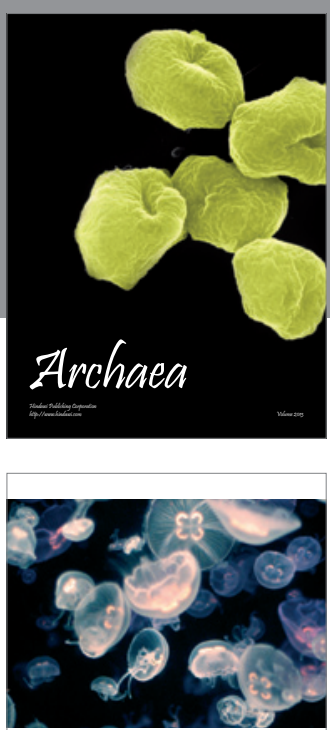

Journal of Marine Biology
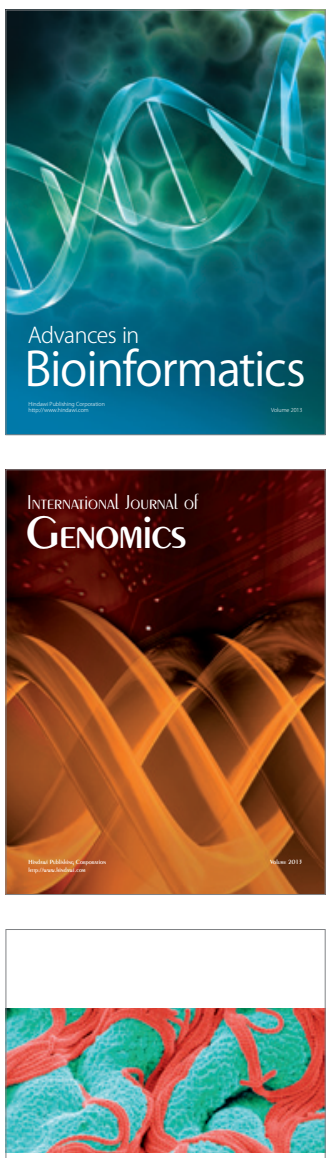

ISRN

Microbiology 